\title{
INFINITARY LATTICE AND RIESZ PROPERTIES OF PSEUDOEFFECT ALGEBRAS AND PO-GROUPS
}

\author{
ANATOLIJ DVUREČENSKIJ and THOMAS VETTERLEIN
}

(Received 26 October 2001; revised 7 August 2002)

\author{
Communicated by B. A. Davey
}

\begin{abstract}
Pseudoeffect (PE-) algebras generalize effect algebras by no longer being necessarily commutative. They are in certain cases representable as the unit interval of a unital po-group, for instance if they fulfil a certain Riesz property.

Several infinitary lattice properties and the countable Riesz interpolation property are studied for PE-algebras on the one hand and for po-groups on the other hand. We establish the exact relationships between the various conditions that are taken into account, and in particular, we examine how properties of a PE-algebra are related to the analogous properties of a representing po-group.
\end{abstract}

2000 Mathematics subject classification: primary 06F05, 81P10; secondary 06F15, 03G12.

Keywords and phrases: pseudoeffect algebras, po-groups, $\sigma$-complete PE-algebras, monotone $\sigma$ complete PE-algebras, orthogonally $\sigma$-complete PE-algebras, PE-algebras with countable Riesz interpolation.

\section{Introduction}

Effect algebras, which play an important role in quantum physics, were introduced to model the set of quantum effects, that is, the set of positive operators of some Hilbert space lying below identity [10]. They were recently generalized to pseudoeffect algebras, or PE-algebras, basically by dropping the axiom of commutativity [5].

The standard effect algebra is the unit interval of the partially ordered group of all self-adjoint operators in Hilbert space, the unit being given by the identity operator. The PE-algebras which are meant to be prototypical arise in an analogous manner; they are given by the unit intervals of po-groups with a strong unit, where these groups are just no longer assumed to be abelian.

(C) 2003 Australian Mathematical Society $1446-7887 / 03 \$ A 2.00+0.00$ 
The question is then natural when a PE-algebra is actually a po-group interval. As a sufficient condition a certain property of Riesz kind has been found in [6]. Moreover, $\mathrm{PE}$-algebras fulfilling this condition and po-groups fulfilling an analogous condition, together with their respective homomorphisms, are categorically equivalent. We shall recall these basic facts in the introductory Section 2 .

Now the relation between such a pair of a PE-algebra of Riesz kind and its representing po-group was already studied in [6], as far as several finitary properties are concerned, that is, lattice order, certain Riesz properties, commutativity and linearity.

In this paper, these studies are continued, and properties are taken into consideration that possibly involve infinitely many elements at a time. So in Section 3, the following properties of PE-algebras, which concern their order, are introduced: completeness, $\sigma$-completeness, and monotone $\sigma$-completeness. Furthermore, we are concerned with orthogonal completeness and orthogonal $\sigma$-completeness. Finally, also the countable Riesz interpolation property is included in the discussion.

In the first step, the exact relations between all these conditions are established under different assumptions; all implications that hold are proved, and for any implication that does not hold, a counterexample is given, which is chosen in a way as simple as possible.

In the second step, in the subsequent Section 4, similar properties are defined for po-groups; in this case, we generalize or recall definitions given elsewhere, in particular in [14] and [12]. Then, also for po-groups, the exact relationships between these conditions are given.

By our third step, we finally arrive at the main scope of this paper. In Section 5, we see how the property of a PE-algebra of Riesz kind is related to the corresponding property of its representing po-group. Namely, we will see (in this order) that countable Riesz interpolation, monotone $\sigma$-completeness, $\sigma$-completeness, orthogonal completeness, orthogonal $\sigma$-completeness, and completeness is preserved from a PE-algebra to the group and vice versa.

\section{PE-algebras and their po-group representation}

Pseudoeffect algebras have been introduced in [5] as partial additive algebras. They have basically the same properties as effect algebras, which have been studied in recent years intensively $[10,4]$. But they differ in one respect: They are no longer commutative. Once commutative, a PE-algebra is the same as an effect algebra. [5] is the basic reference for PE-algebras; their theory is further developed in $[6,7,8]$. The forthcoming paper [9] makes use of some results obtained here.

We recall from [5] that a pseudoeffect algebra, or a PE-algebra for short, is a structure $(E ;+, 0,1)$, where + is a partial binary operation and 0,1 are constants, 
such that, for all $a, b, c \in E$, (PE1) $(a+b)+c$ is defined if and only if $a+(b+c)$ is defined, in which case these elements are equal, (PE2) there is exactly one $d \in E$ and exactly one $e \in E$ such that $a+d=e+a=1$, (PE3) in the case that $a+b$ exists, there are $d, e \in E$ such that $a+b=d+a=b+e$, (PE4) in the case that $1+a$ or $a+1$ exists, we have $a=0$.

A partial order is defined for a PE-algebra $E$ by letting $a \leq b$ if for some $c \in E$ we have $a+c=b$. By (PE3), this order is two-sided: $a \leq b$ holds if and only if for some $d \in E$ we have $d+a=b$.

By (PE2), we may associate to any $a \in E$ a right complement $a^{\sim}$ and a left complement $a^{-}$; that is, we may define unary operations ${ }^{\sim},-$ on $E$ such that

$$
a+a^{\sim}=a^{-}+a=1 .
$$

PE-algebras arise in particular from intervals in partially ordered groups; our axioms are actually designed so as to include this important case. We generalize in this respect again effect algebras; in particular, the standard effect algebra is the unit interval of the abelian po-group of self-adjoint Hilbert space operators.

So let a po-group $G$ and a positive element $u \in G$ be given; in case that $u$ is a strong unit, we call the pair $(G, u)$ a unital po-group. Then $(\Gamma(G, u) ;+, 0, u)$ is a PE-algebra, where $\Gamma(G, u) \stackrel{\text { def }}{=}\left\{g \in G^{+}: g \leq u\right\}$, + is the partial binary operation that is the restriction of the group addition to those pairs of elements of $\Gamma(G, u)$ whose sum lies again in $\Gamma(G, u)$, and 0 is the neutral element of $G$. A PE-algebra $E$ is called an interval PE-algebra if there is a po-group $G$ such that $E \subseteq G^{+}$and $(E ;+, 0,1)=(\Gamma(G, 1) ;+, 0,1)$.

We are interested in the exact algebraic conditions under which a PE-algebra is an interval PE-algebra. This problem, while somewhat difficult to handle in general, gets treatable under a certain condition that may be compared to the Riesz properties known for po-groups. We recall from [5] the following definitions.

We say that a PE-algebra fulfils

$\left(\mathrm{RDP}_{1}\right) \quad$ if for any $a_{1}, a_{2}, b_{1}, b_{2} \in E$ such that $a_{1}+a_{2}=b_{1}+b_{2}$ there are $d_{1}, d_{2}, d_{3}, d_{4} \in E$ such that (i) $d_{1}+d_{2}=a_{1}, d_{3}+d_{4}=a_{2}, d_{1}+d_{3}=b_{1}$, $d_{2}+d_{4}=b_{2}$, and (ii) every $d_{2}^{\prime} \leq d_{2}$ commutes with every $d_{3}^{\prime} \leq d_{3}$;

$\left(\mathrm{RDP}_{0}\right) \quad$ if for any $a, b_{1}, b_{2} \in E$ such that $a \leq b_{1}+b_{2}$ there are $d_{1}, d_{2} \in E$ such that $d_{1} \leq b_{1}, d_{2} \leq b_{2}$ and $a=d_{1}+d_{2}$;

(RIP) if for any $a_{1}, a_{2}, b_{1}, b_{2} \in E$ such that $a_{1}, a_{2} \leq b_{1}, b_{2}$ there is a $c \in E$ such that $a_{1}, a_{2} \leq c \leq b_{1}, b_{2}$.

We note that the following implications hold, and that their converses do not hold [5, Proposition 3.3 (i)]: $\left(\mathrm{RDP}_{1}\right) \rightarrow\left(\mathrm{RDP}_{0}\right) \rightarrow(\mathrm{RIP})$.

Now, the most important result for the sequel is the following [6, Theorem 5.7]. 
THEOREM 2.1. Let $(E ;+, 0,1)$ be a PE-algebra fulfilling $\left(\mathrm{RDP}_{1}\right)$. Then there is an, up to isomorphism unique, unital po-group $(\mathscr{G}(E), 1)$ such that $(E ;+, 0,1)=$ $(\Gamma(G(E), 1) ;+, 0,1)$; that is, $E$ is an interval $P E$-algebra.

REMARK 2.2. For such a pair $E$ and $\mathscr{G}(E)$, we note the following [6, Proposition 6.3]. The order of $E$ as a PE-algebra and the one induced from $\mathscr{G}(E)$ coincide. Moreover, the infimum or supremum of finitely many elements which exists in $E$ also exists in $\mathscr{G}(E)$, and it is the same if calculated in $E$ or in $\mathscr{G}(E)$. Similarly, the infimum or supremum of any subset of $E$ which exists in $\mathscr{G}(E)$ also exists in $E$, and it is the same if calculated in $E$ or in $\mathscr{G}(E)$.

For a PE-algebra $E$ fulfilling $\left(\mathrm{RDP}_{1}\right)$, we will in the sequel refer to $(\mathscr{G}(E), 1)$ simply as the extension of $E$ to its representing unital po-group. It is the relationship between such a pair $E$ and $\mathscr{G}(E)$ which we shall examine in this article.

As a first example of a property preserved from $E$ to $\mathscr{G}(E),\left(\mathrm{RDP}_{1}\right)$ may serve. It is possible to define $\left(\mathrm{RDP}_{1}\right)$ as well as $\left(\mathrm{RDP}_{0}\right)$ and $(\mathrm{RIP})$ for po-groups in exact analogy to PE-algebras; see [5] for explicit definitions. We then have [5, Proposition 4.2 (i)]: $\left(\mathrm{RDP}_{1}\right) \rightarrow\left(\mathrm{RDP}_{0}\right) \leftrightarrow(\mathrm{RIP})$.

Now, we have the following theorem, which contains the statement that $\left(\mathrm{RDP}_{1}\right)$ holds in an interval PE-algebra if and only if it holds in the representing unital po-group [6, Theorem 7.4].

THEOREM 2.3. The map that associates with a PE-algebra E fulfilling $\left(\mathrm{RDP}_{1}\right)$ its representing group $(\mathscr{G}(E), 1)$, defines an equivalence between the category of $P E-$ algebras fulfilling $\left(\mathrm{RDP}_{1}\right)$ together with their homomorphisms and the category of unital po-groups fulfilling $\left(\mathrm{RDP}_{1}\right)$ together with their homomorphisms.

REMARK 2.4. In view of the theorems that will follow, Theorem 2.3 means in particular the following. Anything holding for a pair of a PE-algebra $E$ fulfilling $\left(\mathrm{RDP}_{1}\right)$ and the extension of $E$ to its representing group $(\mathscr{G}(E), 1)$ may be reformulated from the point of view of the theory of partially ordered groups. Namely, Theorem 2.3 implies that we may equivalently assume to be given the pair of a unital po-group $(G, u)$ fulfilling $\left(\mathrm{RDP}_{1}\right)$ and the PE-algebra arising from its unit interval $\Gamma(G, u)=$ $\{g \in G: 0 \leq g \leq u\}$.

Let us continue by exhibiting a certain subclass among the interval PE-algebras as particularly important. We will actually see within the subsequent sections, how our results specialize to this class. Namely, the lattice-ordered PE-algebras fulfilling $\left(\mathrm{RDP}_{1}\right)$ are of special interest, since they may be identified with the pseudo-MV algebras. We refer to [13] for the definition and to [3] for the group representation of pseudo-MV algebras. 
The identification is done in the following simple manner [6]. Given a PE-algebra $(E ;+, 0,1)$ of the mentioned kind, we may extend the partial operation + to a total one, $\oplus$, by defining $a \oplus b \stackrel{\text { def }}{=} a+\left(a^{\sim} \wedge b\right)$ for $a, b \in E$. Then $(E ; \oplus, 0,1,-, \sim)$ is a pseudoMV algebra, and all pseudo-MV algebras arise in this way. From $(E ; \oplus, 0,1,-, \sim)$, we may recover the underlying PE-algebra by defining $a+b \stackrel{\text { def }}{=} a \oplus b$ if $b \leq a^{\sim}$; the operations ${ }^{-}, \sim$ then fulfil (1). The order, defined for pseudo-MV algebras in a similar way as for PE-algebras, is in both cases the same.

In particular, the MV-algebras, which are the commutative pseudo-MV algebras, are thus identifiable with the lattice-ordered commutative PE-algebras fulfilling $\left(\mathrm{RDP}_{1}\right)$.

A pseudo-MV algebra $(E ; \oplus, 0,1,-, \sim)$ is, in accordance with Theorem 2.1, extendable to some unital $\ell$-group $(\mathscr{G}(E), 1)$, as proved in [3]. We have then $\left(E ; \oplus, 0,1,^{-},^{\sim}\right)=\left(\Gamma(\mathscr{G}(E), 1) ; \oplus, 0,1,{ }^{-}, \sim\right)$, where the operations on $\Gamma(\mathscr{G}(E), 1)$ are defined by the $p o$-group operations as follows: $a \oplus b=(a+b) \wedge 1$ and $a^{-}=1-a$, $a^{\sim}=-a+1$ for $a, b \in \Gamma(\mathscr{G}(E), 1)$.

For an MV-algebra, the representing group is abelian, see [17] or [4].

Another subclass of PE-algebras, in this case actually effect algebras, is constructed in a particularly simple manner. This kind of PE-algebra will be used for examples within the subsequent sections. Let $X$ be some set and $E$ a collection of subsets of $X$ such that (i) $E$ contains the empty set, (ii) $E$ is closed under complements, and (iii) for any pairwise disjoint sets $A_{1}, \ldots, A_{n} \in E$ such that $A_{1} \cup \cdots \cup A_{n}=X$, we have $A_{i} \cup A_{j} \in E$ for all $i, j$. Then $(E ; \dot{\cup}, \emptyset, X)$ is a PE-algebra, where $\dot{\cup}$ is defined for a pair of disjoint sets as the set theoretical union whenever this leads to a result in $E$.

To any such a PE-algebra $(E ; \dot{\cup}, \emptyset, X)$, we may associate a representing po-group in the obvious manner. Namely, let $E^{\prime}$ be the set of characteristic functions $\chi_{A}$ of all $A \in E$; and let $G$ be the smallest group of functions $f: X \rightarrow \mathbb{Z}$ which contains $E^{\prime}$. Then we may define $G^{+}$as the subsemigroup of $G$ generated by $E^{\prime}$, since this is easily be seen a positive cone. $(G, 1)$ is then a unital po-group, where 1 is meant to be the constant one function.

We claim that $(E ; \dot{\cup}, \emptyset, X) \cong \Gamma(G, 1)$. It is indeed evident that $\iota: E \rightarrow G, A \mapsto$ $\chi_{A}$ is an injective mapping preserving $\dot{U}$ and the constants. $\iota$ is also surjective; indeed, if $0 \leq f \leq 1$ in $G$, then $f=\chi_{A}$ for some $A \subseteq X$ such that $A=A_{1} \dot{\cup} \ldots \dot{\cup} A_{m}$ and $X \backslash A=A_{1}^{\prime} \dot{\cup} \ldots \dot{\cup} A_{n}^{\prime}$, where $A_{1}, \ldots, A_{m}, A_{1}^{\prime}, \ldots, A_{n}^{\prime} \in E$; by assumption it follows $A \in E$. Moreover, if for $A, B \in E, \iota(A)+\iota(B)$ exists in $\Gamma(G, 1)$, then $\iota(A)+\iota(B)=\chi_{A}+\chi_{B}=\chi_{A \dot{\cup} B}=\iota(A \dot{\cup} B)$.

\section{Infinitary order properties and countable interpolation for PE-algebras}

The fact that certain properties, which may hold in a PE-algebra, are preserved in its representing po-group, has been already shortly mentioned in the preceding 
introductory Section 2. As seen from Theorem 2.3, this applies for example to the Riesz property $\left(\mathrm{RDP}_{1}\right)$; and it is, when we assume $\left(\mathrm{RDP}_{1}\right)$, also true for a latticeordering as well as for commutativity and linearity [6].

The properties that have been taken into account by now were first-order statements and hence involved, so to say, only finitely many elements at a time. The properties discussed in this article may be characterized by involving possibly infinitely many elements at a time, sometimes even subsets of the ground set.

In this section, we consider certain kinds of order completeness and the countable Riesz interpolation for PE-algebras. The first four notions which we will introduce are defined analogously to abelian po-groups; see for example [14] and [12]. The last two notions parallel similar conditions for MV-algebras, proposed in [16].

We shall see first of all how these conditions are interrelated; in the subsequent sections, we will relate them to the analogous properties of po-groups.

DEFINITION 3.1. Let $(E ; 0,+, 0,1)$ be a pseudoeffect algebra.

(i) We say that $E$ is complete, if any subset of $E$ has an infimum and a supremum.

(ii) We say that $E$ is $\sigma$-complete, if any countable subset of $E$ has an infimum and a supremum.

(iii) We say that $E$ is monotone $\sigma$-complete, if any countable set of elements $a_{1}, a_{2}, \ldots \in E$ such that $a_{1} \leq a_{2} \leq \cdots$ possesses a supremum.

(iv) We say that $E$ fulfils the countable Riesz Interpolation Property, or ( $\sigma$-RIP) for short, if for any two countable sets $a_{1}, a_{2}, \ldots, b_{1}, b_{2}, \ldots \in E$ such that $a_{i} \leq b_{j}$ for all $i, j$, there is a $c \in E$ such that $a_{i} \leq c \leq b_{j}$ for all $i, j$.

(v) We say that $E$ is orthogonally complete, if any set $A \subseteq E$ such that $a \wedge b=0$ for any $a, b \in A$ possesses a supremum.

(vi) We say that $E$ is orthogonally $\sigma$-complete, if any countable set $A \subseteq E$ such that $a \wedge b=0$ for any $a, b \in A$ possesses a supremum.

Since the map - on a PE-algebra $E$ is an order-antiautomorphism, we clearly may define $E$ to be monotone $\sigma$-complete equivalently by requiring that every decreasing sequence of elements possesses an infimum.

The interrelation between any pair of conditions among the six ones defined in Definition 3.1, the property of being lattice-ordered, and (RIP), shall be seen now.

PROPOSITION 3.2. Let E be a PE-algebra. The following implications hold.

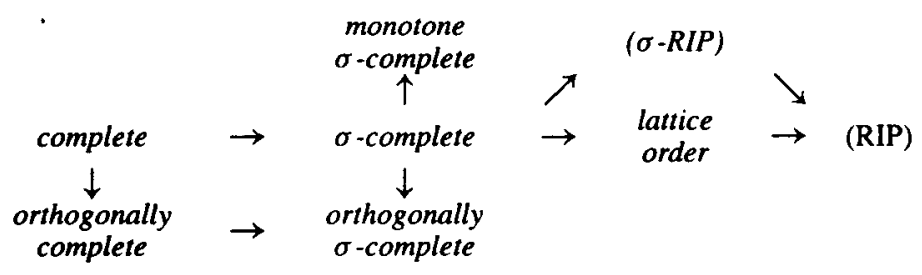


There are no more implications holding in general between any pair of the mentioned conditions than those derivable from the scheme.

PROOF. It is evident that all the implications which are shown in the scheme hold. To exclude that anything more can be shown, the following statements. are sufficient.

From monotone $\sigma$-completeness, neither orthogonal $\sigma$-completeness nor (RIP) follows, as seen from Examples 3.5 and 3.9, respectively.

By $(\sigma$-RIP), neither monotone $\sigma$-completeness, nor orthogonal $\sigma$-completeness, nor lattice ordering is implied, as seen in the first case from Example 3.6 and in the latter two cases from Example 3.5.

From $\sigma$-completeness, orthogonal completeness does not follow, as seen from Example 3.4.

$E$ being lattice ordered, neither implies monotone $\sigma$-completeness, nor ( $\sigma$-RIP), nor orthogonal $\sigma$-completeness, as seen in the first two cases from Examples 3.8 and in the latter case from Example 3.3. Orthogonal completeness does not imply (RIP), as may be seen from Example 3.10.

EXAMPLE 3.3. Let $X$ be a countably infinite set and $E=\{A \subseteq X$ : card $A<$ $\aleph_{0}$ or $\left.\operatorname{card}(X \backslash A)<\aleph_{0}\right\}$. Then $(E ; \dot{\cup}, \emptyset, X)$ is a PE-algebra in the sense of what was noted at the end of Section 2. $E$ is a lattice. Furthermore, $E$ is not orthogonally $\sigma$ complete; the set of singletons from a set $A \subseteq X$ such that card $A=\operatorname{card}(X \backslash A)=\aleph_{0}$ has no supremum.

EXAMPLE 3.4. Let $X$ be an uncountable set and $E=\{A \subseteq X$ : $\operatorname{card} A \leq$ $\aleph_{0}$ or $\left.\operatorname{card}(X \backslash A) \leq \aleph_{0}\right\}$. Then $(E ; \dot{\cup}, \emptyset, X)$ is again a PE-algebra. $E$ is $\sigma$-complete. But $E$ is not orthogonally complete; the set of singletons from a set $A \subseteq X$ such that card $A=\operatorname{card}(X \backslash A)>\aleph_{0}$ has no supremum.

EXAmple 3.5. Let $X$ be a set and $A, B, C$ pairwise disjoint subsets of $X$ such that $X=A \cup B \cup C$; assume further that $\operatorname{card} A=\operatorname{card} B=\operatorname{card} C>\aleph_{0}$. Let $E=\left\{M \Delta N: M=\emptyset, A, A \cup B, B \cup C, C\right.$, or $\left.X ; \operatorname{card} N \leq \aleph_{0}\right\}$. Then $(E ; \dot{\cup}, \emptyset, X)$ is again a PE-algebra. $E$ is monotone $\sigma$-complete and fulfils ( $\sigma$-RIP). But $E$ is no lattice: $A \cup B$ and $B \cup C$ do not have an infimum; $A$ and $C$ do not have a supremum. In particular, $E$ is not orthogonally $\sigma$-complete.

EXAMPLE 3.6. As in the previous example, let $X$ be some set, $A, B, C \subseteq X$ pairwise disjoint such that $X=A \cup B \cup C$, and $\operatorname{card} A=\operatorname{card} B=\operatorname{card} C>\aleph_{0}$. Let now $E=\left\{M \triangle N: M=A, A \cup B, B \cup C\right.$, or $C$ and card $N \leq \aleph_{0} ; \quad$ or $M=$ $\emptyset$ or $X$ and card $\left.N<\aleph_{0}\right\}$. Then the PE-algebra $(E ; \dot{U}, \emptyset, X)$ is no longer monotone $\sigma$-complete, but still fulfils ( $\sigma$-RIP). $E$ is again no lattice, and $E$ is not orthogonally $\sigma$-complete. 
EXAMPLE 3.7. Similarly to the previous examples, let $X$ be some set, $A, B \subseteq X$ disjoint such that $X=A \cup B$, and card $A=\operatorname{card} B>\aleph_{0}$. Let now $E=\{M \triangle N: M=$ $A$ or $B$ and card $N \leq \aleph_{0}$; or $M=\emptyset$ or $X$ and card $N<\aleph_{0}$ ). Then the PE-algebra $(E ; \dot{U}, \emptyset, X)$ is lattice-ordered and fulfils ( $\sigma$-RIP). $E$ is not orthogonally $\sigma$-complete.

EXAMPLE 3.8. Let $E$ be the PE-algebra corresponding to the Chang MV-algebra; compare [4, Example 5.2.39]. So let $E=\{0,1,2,3, \ldots, \hat{3}, \hat{2}, \hat{1}, \hat{0}\}$, and define the addition as follows: For $n, m=0,1,2, \ldots$, let the sum $n+m$ be the usual sum of natural numbers, and let $n+\hat{m}=\hat{m}+n=\widehat{m-n}$ for $n \leq m$.

$E$ is linearly ordered, so in particular lattice-ordered and orthogonally complete. It is not monotone $\sigma$-complete, since $0,1, \ldots$ do not possess a smallest upper bound, and it does not fulfil ( $\sigma$-RIP).

EXAMPLE 3.9. Let $E$ be the standard effect algebra of all self-adjoint operators in Hilbert space lying between 0 and identity. $E$ is monotone $\sigma$-complete; see for example [18, Proposition 4.5.2]. If the dimension of the space is at least 2, then $E$ is not lattice-ordered; the exact condition for a pair of effects that their infimum exists was established in [1], see also [4]. $E$ does not even fulfil (RIP); see for example [5, Example 3.8].

EXAMPLE 3.10. Similarly to the previous example, consider the set $\mathscr{L}(H)_{\text {sa }}$ of all bounded self-adjoint operators in some at least two-dimensional Hilbert space $H$. Define now for a self-adjoint operator $T$ to be strictly positive, or $T \geq_{s} 0$, if it is zero or else bounded away from zero; that is, let

$$
T \geq{ }_{s} 0 \text { if } T=0 \text { or, for some } \epsilon>0, \quad T \geq \varepsilon I,
$$

where $I$ is the identity operator. Let $E=\left\{T \in \mathscr{L}(H)_{\mathrm{sa}}: 0 \leq_{s} T \leq_{s} I\right\}$, and let + be the usual addition of operators whenever this leads to a result in $E$. Then $(E ;+, 0, I)$ is an effect algebra.

Then $E$ is an antilattice, so in particular orthogonally complete. Indeed, assume for some $A, B, C \in E$ that $C=A \wedge_{s} B$. Then we have $C \leq_{s} A$ and $C \leq_{s} B$, and if not $C=A$ or $C=B$, we have for some $\varepsilon>0$ that $C+\varepsilon I \leq A, B$. But this means

$$
\left(C+\frac{\varepsilon}{2} I\right)+\frac{\varepsilon}{2} I \leq A, B \text { and so } C \leq_{s} C+\frac{\varepsilon}{2} I \leq_{s} A, B .
$$

It follows $A \leq_{s} B$ or $B \leq_{s} A$.

But $E$ still does not fulfil (RIP), as seen by a similar argument as in [5, Example 3.8].

When assuming (RIP), the picture changes as follows. 
PROPOSITION 3.11. Let E be a PE-algebra fulfilling (RIP). The following implications hold.

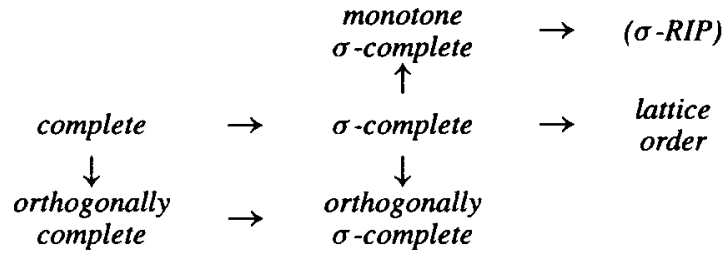

There are no more implications holding in general between any pair of the mentioned conditions than those derivable from the scheme.

ProOF. In view of Proposition 3.2, all we have to show is that from monotone $\sigma$-completeness, ( $\sigma$-RIP) follows. So let $E$ be monotone $\sigma$-complete.

Let $a_{i}, b_{j} \in E, i, j \geq 1$, such that $a_{i} \leq b_{j}$ for all $i, j$. By (RIP) there are $x_{1}, x_{2}, \ldots \in E$ such that

$$
a_{1}, a_{2} \leq x_{1} \leq b_{1}, b_{2}, a_{3}, x_{1} \leq x_{2} \leq b_{1}, b_{2}, a_{4}, x_{2} \leq x_{3} \leq b_{1}, b_{2}, \ldots
$$

Then the set $x_{1}, x_{2}, \ldots$ possesses by assumption a supremum $y_{1}$, and we have $a_{i} \leq$ $y_{1} \leq b_{1}, b_{2}$.

In this manner we may construct a sequence $y_{1}, y_{2}, \ldots \in E$ such that

$$
a_{i} \leq y_{1} \leq b_{1}, b_{2}, a_{i} \leq y_{2} \leq b_{3}, y_{1}, a_{i} \leq y_{3} \leq b_{4}, y_{2}, \ldots \text { for all } i \text {. }
$$

Let then $z$ be the infimum of $y_{1}, y_{2}, \ldots$; it follows $a_{i} \leq z \leq b_{j}$ for all $i, j$.

To see that, in general, no more implication hold than those shown in the scheme, we may in most cases refer to the examples above.

Namely, Example 3.5 shows that monotone $\sigma$-completeness implies neither latticeorder nor orthogonal $\sigma$-completeness. Example 3.6 shows that ( $\sigma$-RIP) does not imply monotone $\sigma$-completeness. Example 3.4 shows that $\sigma$-completeness does not imply orthogonal completeness. Examples 3.8 and 3.3 show that a lattice ordering implies neither $(\sigma$-RIP) nor orthogonal $\sigma$-completeness.

Finally, the subsequent Example 3.12 shows that orthogonal completeness implies neither lattice-order nor ( $\sigma$-RIP).

EXAMPLE 3.12. Let $E=\{(x, y) \in[0,1] \times[0,1]: x=y=0$ or $x=y=$ 1 or $0<x, y<1\}$. Then $(E ;+,(0,0),(1,1))$ is an effect algebra, where + is defined componentwise as the usual addition whenever this leads to result in $E$. Then $E$ is an antilattice, so in particular orthogonally complete. $E$ fulfils (RIP), but $E$ is not a lattice, and $E$ does not fulfil ( $\sigma$-RIP).

We further see how the picture changes when assuming lattice order. 
PROPOSITION 3.13. Let $E$ be a lattice-ordered PE-algebra. The following implications hold.

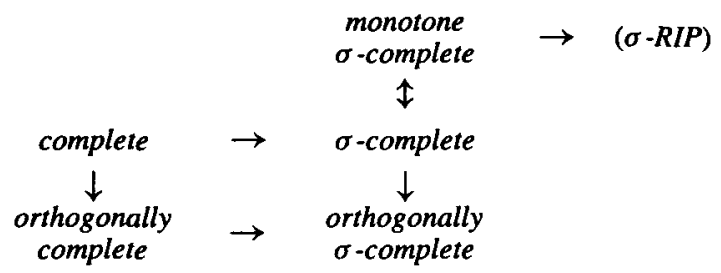

There are no more implications holding in general between any pair of the mentioned conditions than those derivable from the scheme.

PROOF. It is clear that, since $E$ is a lattice, monotone $\sigma$-completeness implies $\sigma$-completeness. The other implications follow from Proposition 3.11.

To see that, in general, no more implication hold than those shown, we may again refer to the examples above.

Namely, Example 3.4 shows that $\sigma$-completeness does not imply orthogonal completeness. Example 3.7 shows that ( $\sigma$-RIP) does not imply orthogonal $\sigma$-completeness. Example 3.8 shows that orthogonal completeness does not imply ( $\sigma$-RIP).

The previous proposition includes in particular pseudo-MV algebras. The notions involved all are purely order-theoretic and thus take over to pseudo-MV algebras in a straightforward manner. We have then in particular that a pseudo-MV algebra is monotone $\sigma$-complete if and only if it is $\sigma$-complete, in which case it fulfils ( $\sigma$-RIP).

It would finally be interesting to see what further happens under the assumption of $\sigma$-completeness. Out of all considered conditions, only two then do not necessarily hold, and we obviously have the following.

PROPOSITION 3.14. Let E be a $\sigma$-complete PE-algebra. The following implication hold.

$$
\text { complete } \rightarrow \quad \begin{gathered}
\text { orthogonally } \\
\text { complete }
\end{gathered}
$$

But in this case, we do not know if the converse of the implication holds in general or not. There might be a connection to the problem if the $\sigma$-completeness of a PEalgebra implies its commutativity, which is discussed to some extent in [9]. In contrast to that, the situation is clear for po-groups; see Proposition 4.2 in the following section.

\section{Infinitary order properties and countable interpolation for po-groups}

The properties discussed in the last section for PE-algebras, are now considered for po-groups. Here, completeness and $\sigma$-completeness are common notions; compare 
for example [2, Chapter XIII]. Monotone $\sigma$-completeness is defined similarly as in [14, Chapter 16]. Countable Riesz interpolation was defined in [12], where it is called $\left(\aleph_{0}, \aleph_{0}\right)$-Interpolation Property. Orthogonal completeness and orthogonal $\sigma$ completeness is defined according to [15].

We note that in the literature often the expression 'Dedekind' or 'conditionally' is attached to the terms introduced in Definition 4.1 (i)-(iii), (v)-(vi), to underline the fact that the statements refer to bounded subsets only. We do not do so in this paper, since we like to have equal names for properties of PE-algebras and of po-groups. Anyhow, without the restriction to bounded sets, the mentioned definitions would not make much sense.

We will see in this section that these conditions are similarly related to each other as in the case of PE-algebras. We discuss in the subsequent section the interrelation of equally denoted properties of PE-algebras on the one side and po-groups on the other side.

Definition 4.1. Let $(G ;+, \leq)$ be a directed po-group. A set $A \subset G$ is called bounded if, for some $a_{l}, a_{u} \in G$, we have $a_{l} \leq a \leq a_{u}$ for all $a \in A$.

(i) We say that $G$ is complete, if any bounded subset of $G$ has an infimum and a supremum.

(ii) We say that $G$ is $\sigma$-complete, if any countable bounded subset of $G$ has an infimum and a supremum.

(iii) We say that $G$ is monotone $\sigma$-complete, if any countable set of elements $a_{1}, a_{2}, \ldots \in G$ such that $a_{1} \leq a_{2} \leq \cdots \leq a$ for some $a \in G$, possesses a supremum.

(iv) We say that $G$ fulfils the countable Riesz Interpolation Property, ( $\sigma$-RIP) for short, if for any two countable sets $a_{1}, a_{2}, \ldots, b_{1}, b_{2}, \ldots \in G$ such that $a_{i} \leq b_{j}$ for all $i, j$, there is a $c \in G$ such that $a_{i} \leq c \leq b_{j}$ for all $i, j$.

(v) We say that $G$ is orthogonally complete, if any bounded set $A \subseteq G^{+}$such that $a \wedge b=0$ for any $a, b \in A$ possesses a suprenium.

(vi) We say that $G$ is orthogonally $\sigma$-complete, if any countable bounded set $A \subseteq G^{+}$such that $a \wedge b=0$ for any $a, b \in A$ possesses a supremum.

Now the six properties just defined, lattice order, and (RIP) are in the case of pogroups related basically in the same way as it was shown for PE-algebras. We will not go into details a second time, but just shortly explain the situation.

The implicational schemes of Propositions 3.2, 3.11, and 3.13 hold also in the case of po-groups. This is in most cases obvious; and in case of Proposition 3.11, the proof is similar; compare also [14, Theorem 16.10].

Moreover, all the counterexamples given in Section 3 are interval PE-algebras. This may be checked in the case of Examples 3.3 to 3.7 by the explanations at the end of Section 2; for Example 3.8, consider the po-group $\mathbb{Z} \times_{\text {lex }} \mathbb{Z}$ with strong unit 
$(1,0)$; for Examples 3.9 and 3.10, consider the group of self-adjoint Hilbert space operators, together with the usual order or the order defined by (2), respectively; and for Example 3.12, consider the group $\mathbb{R} \times \mathbb{R}$ with the strong order and the strong unit $(1,1)$. Now it may be checked directly or with the help of what now follows, that these groups still provide appropriate counterexamples.

Finally, Proposition 3.14 reads for po-groups as follows.

PROPOSITION 4.2. Let $G$ be a $\sigma$-complete po-group. Then the following equivalence holds.

$$
\text { complete } \leftrightarrow \quad \begin{gathered}
\text { orthogonally } \\
\text { complete }
\end{gathered}
$$

PROOF. It is clear that completeness implies orthogonal completeness.

Conversely, a $\sigma$-complete po-group is archimedean (see for example [6, Definition 6.10]) and directed and thus by [11, Chapter V, Corollary 20] abelian. Now, by [15, Theorem 4], an orthogonally complete archimedean abelian $\ell$-group is complete if and only if it is $\sigma$-complete.

\section{Infinitary order properties and countable interpolation for PE-algebras and its representing po-groups}

We shall see in the section how certain of the properties from Definition 3.1 take over from a PE-algebra fulfilling $\left(\mathrm{RDP}_{1}\right)$ to its representing unital po-group.

Let us first consider the properties of fulfilling ( $\sigma$-RIP) and of being monotone $\sigma$-complete. Our results are generalizations of those from [14, Chapter 16] to the non-commutative case.

THEOREM 5.1. Let $(E ;+, 0,1)$ be a PE-algebra fulfilling $\left(\mathrm{RDP}_{1}\right)$, and let $(\mathscr{G}(E), 1)$ be the extension of $E$ to its representing unital po-grcup. Then $E$ fulfils ( $\sigma$-RIP) if and only if so does $\mathscr{G}(E)$.

PROOF. Since the order of $E$ coincides with the one of $\mathscr{G}(E)$, we have that if $\mathscr{G}(E)$ fulfils ( $\sigma$-RIP), so does $E$.

Assume now that $E$ fulfils ( $\sigma$-RIP). We note that, by the remarks of Section 2, $\mathscr{G}(E)$ fulfils $\left(\mathrm{RDP}_{1}\right)$ and consequently also $\left(\mathrm{RDP}_{0}\right)$ and (RIP).

Let now $a_{i}, b_{j} \in \mathscr{G}(E)$ be given such that $a_{i} \leq b_{j}, i, j \geq 1$.

Case 1. Suppose that $0 \leq a_{i} \leq 1$ and $0 \leq b_{j} \leq 2 ; i, j \geq 1$. Then, due to $\left(\mathrm{RDP}_{0}\right), b_{j}=d_{j 1}+d_{j 2}$ for some $d_{j 1}, d_{j 2} \in E$, where $j \geq 1$. By (RIP), there is for any $i, j$ an $e_{i j} \in \mathscr{G}(E)$ such that $a_{i}, d_{j 1} \leq e_{i j} \leq 1, b_{j}$. So for a fixed $j$, we have $d_{j 1}, e_{i j} \leq 1, b_{j}$, an inequality referring entirely to the interval $\left[d_{j 1}, d_{j 1}+1\right]$. Since 
this interval is order-isomorphic to $E$, which fulfils ( $\sigma$-RIP), there is an $f_{j} \in \mathscr{G}(E)$ such that $d_{j 1}, e_{i j} \leq f_{j} \leq 1, b_{j}$ for all $i$. For any $i, j$, we then have $a_{i} \leq f_{j}$. Again by ( $\sigma$-RIP) holding in $E=[0,1]$, there is a $c \in \mathscr{G}(E)$ such that $a_{i} \leq c \leq f_{j} \leq b_{j}$ for any $i, j$.

Case 2. Suppose that $0 \leq a_{i} \leq 2$ and $0 \leq b_{j} \leq 2 ; i, j \geq 1$. Then, due to $\left(\mathrm{RDP}_{0}\right), a_{i}=d_{i 1}+d_{i 2}$ for some $d_{i 1}, d_{i 2} \in E$, where $i \geq 1$. From Case 1 we know that there is an $e \in \mathscr{G}(E)$ such that $d_{i 1} \leq e \leq 1, b_{j}$ for all $i, j$. By (RIP), we may choose for every $i, j$ an $f_{i j} \in \mathscr{G}(E)$ such that $a_{i}, e \leq f_{i j} \leq e+1, b_{j}$. For every $j$, we get again by Case 1 a $g_{j} \in \mathscr{G}(E)$ such that $-e+f_{i j} \leq g_{j} \leq 1,-e+b_{j}$ for all $i$. Now $-1 \leq-g_{j} \leq-f_{i j}+e \leq-a_{i}+e \leq 1$; so once again by Case 1, we get an $h \in \mathscr{G}(E)$ such that $-g_{j}+1 \leq h \leq-a_{i}+e+1$ for all $i, j$. We then have $a_{i} \leq e+1-h \leq e+g_{j} \leq b_{j}$ for any $i, j$.

Case 3. Suppose that for some $v, w \in \mathscr{G}(E)$, we have $v \leq a_{i} \leq w$ and $v \leq b_{j} \leq w$ for all $i, j$. Now the interval $[v, w]$ is order-isomorphic to $[0, w-v]$, which is included in $\left[0,2^{n}\right]$ for some $n \geq 1$. But within this interval, $(\sigma-\mathrm{RIP})$ holds due to Case 2 , which is easily seen by complete induction on $n$.

Case 4. Let finally $a_{i}, b_{j}$ be not necessarily bounded from below or above. By (RIP), there are $x_{1}, x_{2}, \ldots \in \mathscr{G}(E)$ such that

$$
a_{1}, a_{2} \leq x_{1} \leq b_{1}, b_{2}, a_{3}, x_{1} \leq x_{2} \leq b_{1}, b_{2}, a_{4}, x_{2} \leq b_{1}, b_{2}, \ldots
$$

Then we have $x_{1} \leq x_{2} \leq \cdots \leq b_{1}, b_{2}$. Since $\mathscr{G}(E)$ has a strong unit, it is directed; so by Case 3 there is a $y_{1} \in \mathscr{G}(E)$ such that $a_{i} \leq y_{1} \leq b_{1}, b_{2}$ for all $i$.

In this manner we may construct a whole sequence $y_{1}, y_{2}, \ldots \in \mathscr{G}(E)$ such that

$$
a_{i} \leq y_{1} \leq b_{1}, b_{2}, a_{i} \leq y_{2} \leq b_{3}, y_{1}, a_{i} \leq y_{3} \leq b_{4}, y_{2}, \ldots \text { for all } i \text {. }
$$

So we have $a_{i} \leq \cdots \leq y_{2} \leq y_{1}$. By Case 3 , we may construct a sequence $z_{1}, z_{2}, \ldots \in$ $\mathscr{G}(E)$ such that

$$
a_{1}, a_{2} \leq z_{1} \leq y_{j}, a_{3}, z_{1} \leq z_{2} \leq y_{j}, \ldots \text { for all } j
$$

So we have $z_{1} \leq z_{2} \leq \cdots \leq y_{2} \leq y_{1}$, and again by Case 3 there is a $d \in \mathscr{G}(E)$ such that $z_{i} \leq d \leq y_{j}$, which means $a_{i} \leq d \leq b_{j}$, for all $i, j$.

Before turning to a similar theorem concerning monotone $\sigma$-completeness, we prove the following strengthening of Remark 2.2.

PROPOSITION 5.2. Let $(E ;+, 0,1)$ be a PE-algebra fulfilling $\left(\mathrm{RDP}_{1}\right)$ and $(\sigma-\mathrm{RIP})$, and let $(\mathscr{G}(E), 1)$ be the extension of $E$ to its representing unital po-group. Then countable infima and suprema are preserved from $E$ to $\mathscr{G}(E)$. 
ProOF. By Theorem 5.1 we know that $\mathscr{G}(E)$ fulfils ( $\sigma$-RIP).

Let $a, a_{1}, a_{2}, \ldots \in E$, and $a=\bigwedge_{i} a_{i}$ in $E$. Then $a \leq a_{i}$ in $E$ and so in $\mathscr{G}(E)$ for all $i$. Now if $a^{\prime} \in \mathscr{G}(E)$ and $a^{\prime} \leq a_{i}$ for every $i$, there is by ( $\sigma$-RIP), holding in $\mathscr{G}(E)$, a $b \in \mathscr{G}(E)$ such that $a^{\prime}, 0 \leq b \leq a_{i}$ for every $i$. Since then $b \leq a_{i}$ also in $E$, we have $b \leq a$ in $E$ as well as in $\mathscr{G}(E)$; so it follows $a^{\prime} \leq a$ in $\mathscr{G}(E)$. So we showed that countable infima are preserved from $E$ to $\mathscr{G}(E)$.

Analogously, we see that also countable suprema are preserved.

THEOREM 5.3. Let $(E ;+, 0,1)$ be a PE-algebra fulfilling $\left(\mathrm{RDP}_{1}\right)$, and let $(\mathscr{G}(E), 1)$ be the extension of $E$ to its representing unital po-group. Then $E$ is monotone $\sigma$ complete if and only if so is $\mathscr{G}(E)$.

ProOF. In view of Remark 2.2, we have that if $\mathscr{G}(E)$ is monotone $\sigma$-complete, so is $E$.

Let now $E$ be monotone $\sigma$-complete. Since by [5, Proposition 3.3 (i)] $E$ fulfils (RIP), $E$ fulfils by Proposition 3.11 also ( $\sigma$-RIP). From this we conclude by Theorem 5.1 that $\mathscr{G}(E)$ fulfils $\left(\sigma\right.$-RIP). Furthermore, we note again that $\mathscr{G}(E)$ fulfils $\left(\mathrm{RDP}_{1}\right)$, $\left(\mathrm{RDP}_{0}\right)$ and (RIP).

We now show that every decreasing sequence in $[0,2]$ possesses an infimum. From that, we may conclude by complete induction that the same is true for the intervals $\left[0,2^{n}\right], n \geq 1$, by which fact the monotone $\sigma$-completeness follows.

So let $a_{1}, a_{2}, \ldots \in \mathscr{G}(E)$ be given such that $0 \leq \cdots \leq a_{2} \leq a_{1} \leq 2$. By (RIP) there are elements $d_{i 1}, d_{i 2} \in E, i \geq 1$, such that

$$
\begin{aligned}
& a_{1}=d_{11}+d_{12}, \\
& a_{2}=d_{21}+d_{22},
\end{aligned}
$$

By the monotone $\sigma$-completeness holding in $E$, the infima $d_{1}=\bigwedge_{i} d_{i 1}$ and $d_{2}=\bigwedge_{i} d_{i 2}$ exist, and by Proposition 5.2, these equation hold also in $\mathscr{G}(E)$. We then have

$$
d_{1}+d_{2}=\bigwedge_{i} d_{i 1}+\bigwedge_{j} d_{j 2}=\bigwedge_{i}\left(d_{i 1}+\bigwedge_{j} d_{j 2}\right)=\bigwedge_{i} \bigwedge_{j}\left(d_{i 1}+d_{j 2}\right)=\bigwedge_{i, j}\left(d_{i 1}+d_{j 2}\right) .
$$

Since for any pair $i, j$ we have $d_{k 1}+d_{k 2} \leq d_{i 1}+d_{j 2}$, where $k=\max \{i, j\}$, we conclude $d_{1}+d_{2}=\bigwedge_{i}\left(d_{i 1}+d_{i 2}\right)=\bigwedge_{i} a_{i}$.

We note that for $\mathrm{PE}$-algebras fulfilling $\left(\mathrm{RDP}_{1}\right)$, monotone $\sigma$-completeness actually implies commutativity. Indeed, a monotone $\sigma$-complete unital po-group is directed and archimedean and thus commutative; we refer to the forthcoming paper [9] for the details. 
THEOREM 5.4. Let $(E ;+, 0,1)$ be a PE-algebra fulfilling $\left(\mathrm{RDP}_{1}\right)$, and let $(\mathscr{G}(E), 1)$ be the extension of $E$ to its representing unital po-group. Then $E$ is $\sigma$-complete if and only if so is $\mathscr{G}(E)$.

PROOF. In view of Remark 2.2, we have that if $\mathscr{G}(E)$ is monotone $\sigma$-complete, so is $E$.

Conversely, let $E$ be $\sigma$-complete. Then $E$ is monotone $\sigma$-complete, and so, by Theorem 5.3, $\mathscr{G}(E)$ is monotone $\sigma$-complete. Furthermore, $E$ is lattice-ordered, and so, by [6, Proposition 6.4], $\mathscr{G}(E)$ is an $\ell$-group. It follows that $\mathscr{G}(E)$ is $\sigma$-complete.

In the following way, Theorems 5.1, 5.3, and 5.4 specialize to pseudo-MV algebras.

THEOREM 5.5. Let $E$ be a pseudo-MV algebra, and let $(\mathscr{G}(E), 1)$ be the extension of $E$ to its representing unital $\ell$-group. Then $E$ fulfils ( $\sigma$-RIP) if and only if so does $\mathscr{G}(E)$. Moreover, $E$ is monotone $\sigma$-complete if and only if $E$ is $\sigma$-complete if and only if $\mathscr{G}(E)$ is monotone $\sigma$-complete if and only if $\mathscr{G}(E)$ is $\sigma$-complete.

We will now proceed with the properties of orthogonal completeness and orthogonal $\sigma$-completeness.

THEOREM 5.6. Let $(E ;+, 0,1)$ be a PE-algebra fulfilling $\left(\mathrm{RDP}_{1}\right)$, and let $(\mathscr{G}(E), 1)$ be the extension of $E$ to its representing unital po-group. Then $E$ is orthogonally complete if and only if so is $\mathscr{G}(E)$.

Furthermore, $E$ is orthogonally $\sigma$-complete if and only if so is $\mathscr{G}(E)$.

PROOF. We prove the first part only; for the second one, we may proceed similarly.

So let $\mathscr{G}(E)$ be orthogonally complete. If then any pair of distinct elements of some set $A \subseteq E$ has infimum 0 , the same is by Remark 2.2 true with respect to $\mathscr{G}(E)$. Hence $A$ has a supremum in $\mathscr{G}(E)$, which again by Remark 2.2 is the supremum also with respect to $E$. So $E$ is orthogonally complete.

Conversely, let $E$ be orthogonally complete. Let $\left\{a_{\imath}: \imath \in I\right\} \subseteq \mathscr{G}(E)^{+}$be a bounded set such that $a_{t_{1}} \wedge a_{t_{2}}=0$ whenever $\iota_{1} \neq \iota_{2}$. We have to show that $\bigvee_{1} a_{t}$ exists in $\mathscr{G}(E)$.

Step 1. Assume first that $a_{\imath} \leq 1$, that is, $a_{\imath} \in E$, for all $\iota$. Then $a=V_{\imath} a_{t}$ exists in $E$; we have to show that $a$ is the supremum of the $a_{t}$ also in $\mathscr{G}(E)$. Clearly, $a_{\imath} \leq a$ holds also in $\mathscr{G}(E)$; let now $b \in \mathscr{G}(E)$ such that $a_{\iota} \leq b$ for all $\iota$. By $\left(\mathrm{RDP}_{0}\right)$, there are $b_{1}, \ldots, b_{n} \in E$ such that $b=b_{1}+\cdots+b_{n}$ for some $n$; we may assume $n \geq 2$. Furthermore, for every $\iota$, there are $a_{t 1} \leq b_{1}, \ldots, a_{t n} \leq b_{n}$ in $E$ such that $a_{\imath}=a_{t 1}+\cdots+a_{t n}$. Now by assumption, the suprema $a^{1}=\bigvee_{\imath} a_{t 1}, \ldots, a^{n}=\bigvee_{\imath} a_{\imath n}$ exist in $E$. We have then in $E$, and consequently in $\mathscr{G}(E), a^{1} \leq b_{1}, \ldots, a^{n} \leq b_{n}$. 
Now, in $E$, the sum $a_{t_{1} 1}+a_{t_{2} 2}$ exists for all pairs of indices $\iota_{1}, \iota_{2} \in I$; for this is true by construction if they are equal, and otherwise the two elements have zero infimum, whence their sum exists and equals $a_{t 1} \vee a_{t 22}$ by [5, Lemma 3.2 (ii)]. It follows in particular that $a_{\imath 11} \leq a_{t_{2}}^{-}$for all $\iota_{1}, \iota_{2}$, so $\bigvee_{\imath} a_{\imath 1} \leq \bigwedge_{\imath} a_{t 2}^{-}=\left(\bigvee_{1} a_{t 2}\right)^{-}$, whence $a^{1}+a^{2}$ exists. Using these facts and [5, Remark 1.8], we may calculate in $E$

$$
a^{1}+a^{2}=\bigvee_{\imath} a_{t 1}+\bigvee_{\imath} a_{t 2}=\bigvee_{t_{1}, \iota_{2}}\left(a_{t 1}+a_{t 2}\right)=\bigvee_{\imath}\left(a_{t 1}+a_{t 2}\right)
$$

By an inductive argument, we see in a similar manner that

$$
a^{1}+\cdots+a^{n}=\bigvee_{\imath}\left(a_{t 1}+\cdots+a_{t n}\right)=\bigvee_{\imath} a_{t}=a
$$

So in $\mathscr{G}(E)$, we have $a=a^{1}+\cdots+a^{n} \leq b_{1}+\cdots+b_{n}=b$, which finishes the proof that $a=V_{i} a_{i}$ in $\mathscr{G}(E)$.

Step 2. Assume now the general case; then for some multiple $k$ of the strong unit 1 , we have $a_{t} \leq k$ for all $t$, and we may write $a_{t}=a_{t 1}+\cdots+a_{t k}$ for certain $a_{t 1}, \ldots, a_{t k} \in E$. By assumption, the suprema $\bigvee_{\imath} a_{t 1}, \ldots, \bigvee_{1} a_{t k}$ exist in $E$ and by Step 1 also in $\mathscr{G}(E)$; so we have in $\mathscr{G}(E)$

$$
\bigvee_{\imath} a_{t 1}+\cdots+\bigvee_{\imath} a_{t k}=\bigvee_{\imath_{1} \ldots, \ldots, t_{k}}\left(a_{t 11}+\cdots+a_{t k}\right)
$$

Now the sum of elements of a po-group whose infimum is 0 equals their supremum; this is why we may, similarly as above, leave out in (3) all sums in which some pair of summands has infimum 0 . We conclude

$$
\bigvee_{\imath} a_{t 1}+\cdots+\bigvee_{\mathfrak{l}} a_{t k}=\bigvee_{\mathfrak{l}}\left(a_{t 1}+\cdots+a_{t k}\right)=\bigvee_{\imath} a_{\imath}
$$

and we have proved that $\mathscr{G}(E)$ is orthogonally complete.

We finally turn to the property of completeness.

THEOREM 5.7. Let $(E ;+, 0,1)$ be a PE-algebra fulfilling $\left(\operatorname{RDP}_{1}\right)$, and let $(\mathscr{G}(E), 1)$ be the extension of $E$ to its representing unital po-group. Then $E$ is complete if and only if so is $\mathscr{G}(E)$.

Proof. In view of Remark 2.2, we have that if $\mathscr{G}(E)$ is complete, so is $E$.

Conversely, assume $E$ to be complete. Then in particular, $E$ is $\sigma$-complete and orthogonally complete. By Theorems 5.4 and 5.6, also $\mathscr{G}(E)$ is $\sigma$-complete and orthogonally complete. By Proposition 4.2, $\mathscr{G}(E)$ is complete. 


\section{Acknowledgement}

The paper has been supported by the grant VEGA 2/7193/20 SAV, Bratislava.

\section{References}

[1] T. Ando, 'Problem of infimum in the positive cone', in: Geometric inequalities and applications (eds. T. M. Rassias and H. M. Srivastava) (Kluwer, Dordrecht, 1999) pp. 1-12.

[2] G. Birkhoff, Lattice theory, Colloquium Publications 25, 3rd Edition (Amer. Math. Soc., Providence, 1995).

[3] A. Dvurečenskij, 'Pseudo-MV algebras are intervals in $\ell$-groups', J. Aust. Math. Soc. 72 (2002), $427-445$.

[4] A. Dvurečenskij and S. Pulmannová, New trends in quantum structures (Kluwer, Dordrecht, 2000).

[5] A. Dvureð̌enskij and T. Vetterlein, 'Pseudoeffect algebras. I. Basic properties', Internat. J. Theoret. Phys. 40 (2001), 685-701.

[6] - 'Pseudeffect algebras. II. Group representations', Internat. J. Theoret. Phys. 40 (2001), 703-726.

[7] — 'Congruences and states on pseudo-effect algebras', Found. Phys. Letters 14 (2001), 425446.

[8] — ' 'On pseudoeffect algebras which can be covered by pseudo MV-algebras', Demonstratio Math. 36 (2003), 261-282.

[9] — 'Archimedeanness and the McNeille completion of pseudoeffect algebras and po-groups', Algebra Universalis, to appear.

[10] D. J. Foulis and M. K. Bennett, 'Effect algebras and unsharp quantum logics', Found. Phys. 24 (1994), 1325-1346.

[11] L. Fuchs, Partially ordered algebraic systems (Pergamon Press, Oxford, 1963).

[12] — ' 'Riesz groups', Annali della Scuola Norm. Sup. Pisa, III. Ser. 19 (1965), 1-34.

[13] G. Georgescu and A. Iorgulescu, 'Pseudo-MV algebras', Mult.-Valued Log. 6 (2001), 95-135.

[14] K. R. Goodearl, Partially ordered abelian groups with interpolation, Mathematical Surveys and Monographs 20 (Amer. Math. Soc., Providence, 1986).

[15] J. Jakubik, 'Conditionally orthogonally complete $\ell$-groups', Math. Nachr. 65 (1975), 153-162.

[16] —_, 'On archimedean MV-algebras', Czechoslovak Math. J. 48 (1998), 575-582.

[17] D. Mundici, 'Interpretation of AF $C^{*}$-algebras in Łukasiewicz sentential calculus', J. Funct. Anal. 65 (1986), 15-63.

[18] G. K. Pedersen, Analysis now (Springer, New York, 1989).

Mathematical Institute

Slovak Academy of Sciences

Štefánikova 49

SK - 81473 Bratislava

Slovakia

e-mail: dvurecen@mat.savba.sk, vetterl@mat.savba.sk 
